\title{
A Comparison of Scent-Delivery Devices and Their Meaningful Use for In-Car Olfactory Interaction
}

\author{
Dmitrijs Dmitrenko, Chi Thanh Vi, Marianna Obrist \\ SCHI Lab, Creative Technology Research Group, School of Engineering and Informatics \\ University of Sussex, Chichester I, Falmer, Brighton, BN1 9QJ, UK \\ \{d.dmitrenko, c.vi, m.obrist\}@ sussex.ac.uk
}

\begin{abstract}
In the field of Human Computer Interaction (HCI), vision and audition have been the dominating modalities for interacting with users. This is despite the fact that humans are equipped with five basic senses. Because of this, there is a limited number of tools that harness the olfactory system as a communication channel. Recently, several promising scent-delivery devices have been developed, however, there is a lack of guidance on how to use them in a meaningful way for different interactive tasks. In this paper, we propose a three-dimensional framework to compare different scent-delivery devices based on the distance, volume, and speed of the scent-delivery. We discuss how this initial exploration can guide the design of in-car olfactory interfaces beyond previous work on drivers' physical and emotional state.
\end{abstract}

\section{ACM Classification Keywords}

H.5.m Information interfaces and presentation (e.g., HCI); H.1.2 User/Machine Systems: Human factor.

\section{Author Keywords}

Comparison framework; olfactory experiences; scent-delivery devices.

\section{INTRODUCTION AND BACKGROUND}

In recent years, there has been an increased interest in smellbased interaction design and advancements in scent-delivery devices for the end user market [7]. Consequently, the use of olfactory stimulation has gained attention in the automotive context. For example, Yoshida et al. [14] developed a system to fight drowsiness while driving and has demonstrated in a study that releasing specific smells (peppermint, rosemary, eucalyptus and lemon) could extend the wakefulness of the driver. Baron and Kalsher [1] proved that the scent of lemon increases both alertness and the mood of the driver, while Martin and Cooper [6] showed it to have a positive impact on people's braking performance during a simulated driving task. Moreover, Raudenbush et al. [9] demonstrated

Automotive'UI 16, October 24-26, 2016, Ann Arbor, MI, USA (c) 2016 ACM. ISBN 978-1-4503-4533-0/16/10 . \$ $\$ 15.00$. DOI: http://dx.doi.org/10.1145/3003715.3005464 an increase in driver's alertness and attentiveness through the release of peppermint and cinnamon. While it was shown that both peppermint and cinnamon reduced frustration and helped participants to focus on the driving task, peppermint was also associated with faster reaction times.

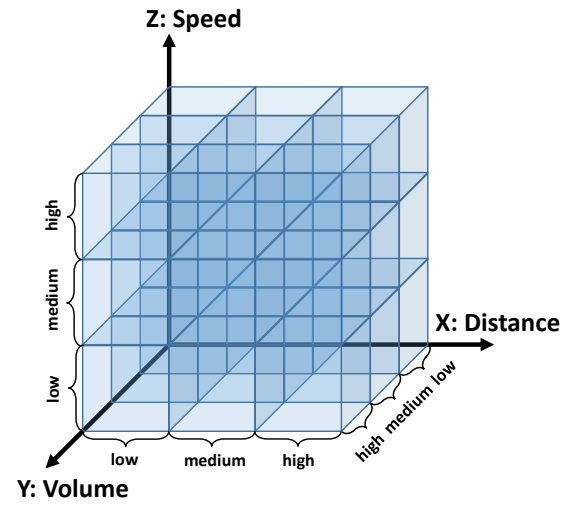

Figure 1: Three-dimensional comparison framework used to evaluate the X: Distance, Y: Volume, and Z: Speed of scentdelivery demonstrated by four commercially available devices.

All these previous studies demonstrate the potential to enhance the in-car interaction. However, these findings are mainly focused on the driver and the primary task of driving, but do not go beyond the modulation of their physical (e.g. fighting drowsiness, increasing alertness) and emotional state (e.g. better mood when driving). We aim to extend this emerging field of research and are interested in understanding to what extent smell stimulation can be used to convey specific information in the automotive context. Smell could be used to convey information both to the driver and to the passengers (co-driver and rear seat passengers). Some examples for this could be as follows: (i) the driver receives a scented notification of approaching a point of interest (e.g. gas station), (ii) the co-driver receives a smell for an interesting landmark recommended by Google Maps, (iii) rear-seat passengers receive different scents to enhance the interactive experience when watching movies or playing computer games. Previous work outside the automotive context has shown that smell can enhance specific activities and create new experiences. Brewster et al. [3] demonstrated the use of a smell-based photo tagging tool in their Olfoto prototype, while Bodnar et. al. [2] studied the use of smell as an ambient notification modality. 


\begin{tabular}{rcccc} 
& \multicolumn{4}{c}{ Scent-Delivery Devices: Manufacturer Specifications } \\
\cline { 2 - 5 } Characteristics & Vortex Activ USB & Scentee & oPhone DUO & Aroma Shooter \\
\hline Scent cartridges & 4 & 1 & 8 & 6 \\
Scent combinations & 16 & 0 & $>300000$ & 64 \\
Platforms & Windows & iOS & iOS & Windows/Linux/iOS \\
Interfaces & USB & Audio Output & Bluetooth & USB \\
\hline
\end{tabular}

Table 1: Comparison of four scent-delivery devices based on their key features.

Their results indicate that smell is less disruptive than visual and auditory stimuli. In addition, Herz and Engen [4] pointed out that smell provides more vivid experiences than any other sense, proving itself as a very powerful interaction medium.

Despite all the advancements in this field, we are still left with the question of which parameters of a scent-delivery device we should pay attention to when tackling the challenge of conveying specific information using smell. As all devices are unique, we established a framework for the comparison of all scent-delivery devices, using three dimensions: distance, volume, and speed of scent-delivery (see Figure 1). These three dimensions are relevant comparison criteria across devices and provide a starting point for a meaningful design exploration of in-car olfactory interaction. We will first present the comparison framework and then apply it to compare four commercially available scent-delivery devices. We will conclude with the discussion of how to apply these devices to in-car interaction scenarios based on smell.

\section{COMPARISON FRAMEWORK}

We compared four commercially available scent-delivery devices: (i) Vortex Activ USB ${ }^{1}$, (ii) Scentee ${ }^{2}$, (iii) oPhone $\mathrm{DUO}^{3}$, and (iv) Aroma Shooter ${ }^{4}$. Each device has specific characteristics with respect to the number of scent cartridges, scent combinations, interaction possibilities (interfaces and control software), and platform compatibility issues (Table 1).

It is worth noting that this was not a quantitative experimental study (which is planned for future work), but a first qualitative exploration to capture the core characteristics and abilities of the four devices enabling us to map them into the 3D space offered by our framework. The first author of this paper has tested each of the four devices individually in a lab environment and recorded the particular delivery pathways and experiences. These were then discussed with the co-authors. A stopwatch and a ruler were used to record time (used to calculate the speed) and distance. The volume of scent-delivery was estimated based on the perceivability of the delivered scent in different locations around the device. Different scents were used in the testing process. Based on this comparison, we wanted to understand the potential of the explored devices in the context of smell-based in-car interaction scenarios. Our comparison framework is based on 3 dimensions (Figure 1):

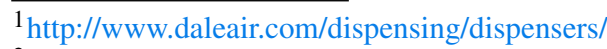

${ }^{2} \mathrm{http}: / / \mathrm{www}$. scentee.com/

${ }^{3} \mathrm{http}: / /$ www.indiegogo.com/projects/ophone-duo/

${ }^{4}$ http://www.aromajoin.com/
}

1. Distance: measured between the device and the furthest point at which the smell is still clearly perceived by the user. Example units to measure are centimetres, metres, etc.

2. Volume: the size of space that a smell can expand in and still be clearly distinguished by the user. Example units to measure are cubic centimetres, cubic metres, etc.

3. Speed: the average speed across the distance dimension. Example units are $\mathrm{cm} / \mathrm{s}, \mathrm{m} / \mathrm{s}$, etc.

As a first step, we mapped each of the four devices onto a two-dimensional space, where two of the three proposed dimensions form the two axes. As we aim to present the framework, rather than precise measurements in this paper, we categorised the devices into three levels of each dimension: low, medium, and high (see Figure 2). We made a decision on which level to map each device to, based on our observations, comparing the devices with each other. The nature of such comparison suggests no strict baseline, which still needs to be established in the future.
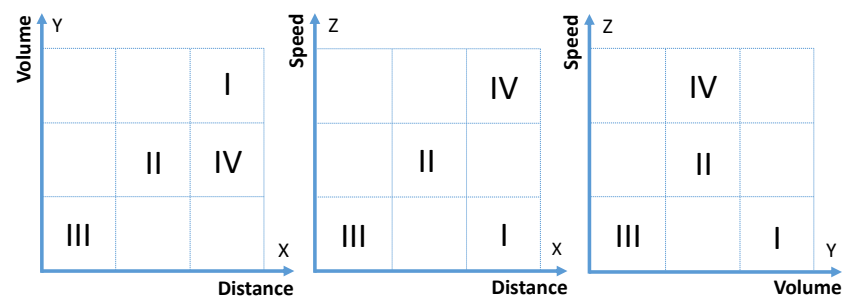

Figure 2: Mapping of the devices on the 2D spaces derived from the $3 \mathrm{D}$ comparison framework, by comparing the corresponding dimensions of the following four devices to each other: (I) Vortex Activ USB, (II) Scentee, (III) oPhone DUO, (IV) Aroma Shooter.

The benefit of mapping the devices on a 2D space before projecting them onto our 3D framework are two-fold. First, two dimensions allow an easy evaluation of a new scent-delivery device, which can then be further projected into a 3D space. Second, the 2D spaces are useful to explore different interaction scenarios, where one of the dimensions might be irrelevant, and hence, an HCI designer only needs to work on an ad-hoc and simplified version for the comparison. For example, speed might not be relevant, when the scent-delivery device is needed for non-urgent notifications (e.g. approaching the destination, when the journey is very long). 

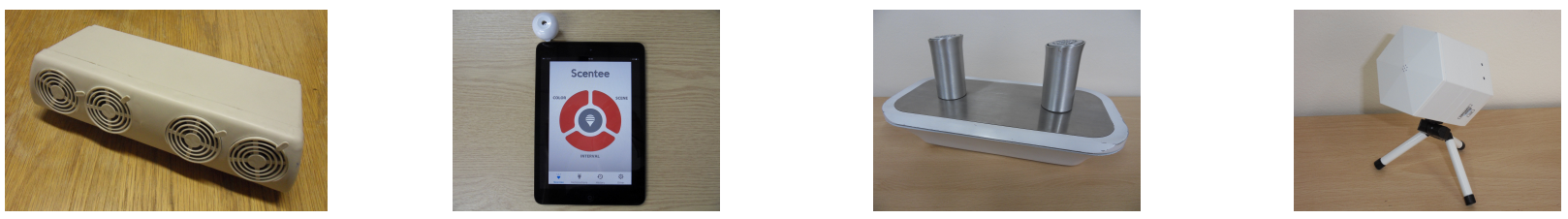

Figure 3: Scent-delivery devices (from left to right): Vortex Activ USB, Scentee on an iPad, oPhone DUO, Aroma Shooter.

\section{Device I: Vortex Activ USB}

Vortex Activ USB (Figure 3) is a scent dispensing system developed by DaleAir that allows the delivery of four individual scents by exposing their cartridges to four individually controlled fans. Over 300 different scent cartridges are available for purchase. Each scent cartridge is located in a separate tube inside the device. Once the driver is installed (automatically under MS Windows), this device can be considered a plugand-play USB controller. Four scents can be released either individually or in combination, by turning the fans on or off in the graphical user interface (no further features are available to control the intensity or duration). The smell released by this device was perceived $5 \mathrm{~m}$ away from the output in any direction after one minute, and we assigned the following dimensions: distance - high, volume - high, speed - low.

\section{Device II: Scentee}

Scentee is a scent-based extension for iOS devices. It comes in the form of a scent cartridge that needs to be charged and then plugged into the audio output of an iPhone/iPad. A variety of scent cartridges can be purchased, but only one at a time can be used. The scent extension is controlled by the Scentee app, allowing the user to manipulate the duration and the interval of the scent delivery (Figure 3 ). Scentee pushes the odorized air out of the device in the shape of a $10-15 \mathrm{~cm}$ long misted cloud. This increases the detectability of the released scent which is further indicated by a blinking LED light. However, the user needs to hold their nose close to the release point in order to perceive the scent. Using Scentee, the odorized air reaches the user's nose approximately 2 seconds after the release. We assigned the following dimensions: distance medium, volume - medium, speed - medium.

\section{Device III: oPhone DUO}

oPhone DUO by Vapor Communications comes with eight scent cartridges, and is controlled by the oNotes iOS app. The interface of the oPhone requires the user to move the nose closer to the scent releasing unit through the design of two tubes (see Figure 3), each containing four different scents, which can be further mixed. The scent can be perceived at a distance of not more than $10 \mathrm{~cm}$, in approximately $5 \mathrm{sec}-$ onds. We assigned it the following dimensions: distance - low, volume - low, speed - low.

\section{Device IV: Aroma Shooter}

The Aroma Shooter (Figure 3) was developed by Aromajoin and gives space for 6 scent cartridges. The device works across platforms and is connected through USB. This device delivers a directional airflow, allowing precise transmission of scent at a distance of up to $30 \mathrm{~cm}$ after 3 seconds. The delivered scent can be easily detected by a person at that distance, however if the nose is displaced or a person is moving their head, the scent-delivery could be missed. This can be compensated for by a longer delivery time, which can be set in milliseconds using the API. The developer can also choose which of the 6 scents to deliver, to what extent the air blower is activated, and the desired intensity of the scent. We assigned the following dimensions: distance - high, volume - medium, speed - high.

\section{DISCUSSION FOR THE AUTOMOTIVE CONTEXT}

In this paper, we propose a comparison framework based on the exploration of four devices. We compared the devices with respect to distance, volume, and speed of the scent-delivery in the first step. We are aware that there are many more features that could have been considered and included in the comparison (e.g. size of the device, type of the actuators and created airflow, physical and chemical properties of the odorants). However, from an interaction design perspective, distance, volume, and speed form a good starting point, as they can be linked to specific tasks, scenarios, and interaction goals. Below we discuss all the investigated devices in relation to various in-car interaction scenarios based on smell.

Due to its directional stimulation, the Aroma Shooter is very suitable for precise scent delivery and quick reaction times. Such a mechanism could become very beneficial for the interaction of the driver with the car, extending existing audiovisual modalities by smell, through a crossmodal design approach, such as described by Pfleging et al. [8]. Alternatively it could be applied to deliver a feedforward stimulus for drivingtake-over tasks in (semi)autonomous driving, currently limited to audio input such as investigated by Koo et al. [5].

The user can easily interact with Scentee while on the move. This device is suitable for mobile applications, where it is desirable to keep the scent-delivery within a personal space and not to disturb surrounding people. Hence, it makes it suitable for the co-driver, who can take the eyes away from the road and interact with a mobile device. Scentee could be used to receive notifications from social media or to indicate interesting landmarks in the environment (e.g. retrieved from Tripadvisor). This device could also be used for the stimulation of the co-driver to modulate positive judgment of driver's performance when using a parking assistant, extending previous work in this field carried out by Trösterer et al. [11].

Similarly to Scentee, oPhone DUO is suitable for the delivery of less urgent information, but is less portable and currently only useful for desktop applications. It could potentially be integrated into the rear seat of the car. We see its application in the delivery of scents as rewards for children playing games in the back of a car, extending the scenarios proposed by 
Sundström et al. [10]. The benefit of the oPhone DUO is its high number of scent combinations, which opens opportunities for a broader design spectrum and more interactive use cases. It is also important to mention that a new smaller version of the oPhone, for the car context, has been announced ${ }^{5}$. This device may have applications beyond the rear seat scenarios.

Finally, the Vortex Activ USB device is suitable for ambient uses, such as influencing the mood and emotions. Its application for interaction tasks relies on several constraints. The separation of scent cartridges proved itself not suitable for quick changes between different smells. Compared to other devices, Vortex Activ USB also offers a less sophisticated control over the delivery parameters, but it is useful for ambient notification, such as suggested by Warnock et al. [12]. This device could be applied to give ambient warnings ahead of time (e.g. for upcoming traffic jams), or to send notifications relevant to all the people seated inside the car (e.g. to inform about the remaining traveling time retrieved from the navigation system).

\section{CONCLUSIONS AND DIRECTIONS FOR FUTURE WORK}

We presented four different scent-delivery devices currently available on the market, with respect to their scent-delivery potential based on distance, volume, and speed. In Figure 4 we mapped the explored devices based on the chosen parameters, but a further investigation to grasp the differences between olfactory stimulation opportunities is still needed.

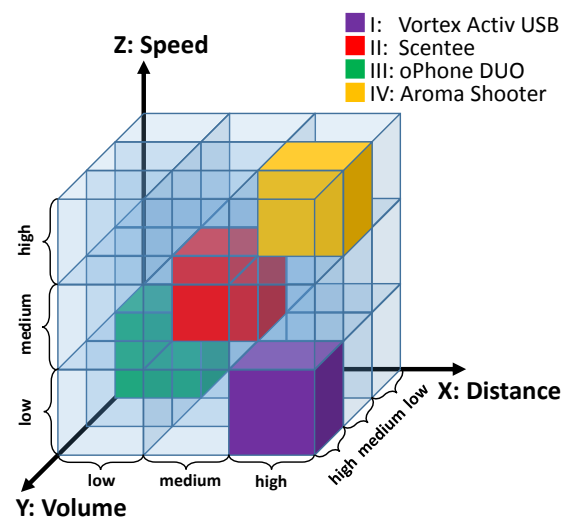

Figure 4: A 3D evaluation framework with four scent-delivery devices mapped onto it.

In the scope of the in-car interface design, scents could be released as alarming or rewarding stimuli, enhance the quality of driving experience and the pleasure of driving, or provide added value for the in-car infotainment. Here it will be crucial to explore controllability and extendibility of the sensations, as well as types of aromas (liquid or solid). These parameters will offer new dimensions for the comparison of such devices. For the future work, it is essential to systematically explore the various devices and how they can be useful and meaningful for in-car interactions based on smell. In particular, we plan to investigate the use of the Aroma Shooter for car-driver

\footnotetext{
${ }^{5}$ http://www.newyorker.com/tech/elements/is-digital-smell-doomed
}

interaction, Scentee for the infotainment of the co-driver, and oPhone for rear seat applications. Vortex Activ USB could be applied for non-urgent notifications of the driver and/or passengers. Moreover, another big challenge is to not just deliver, but also to control the scent-delivery, as well as remove the smell from an enclosed space, such as the interior of a car. Weiss et al. [13] proposed "olfactory white", which might provide a way towards increasing the meaningful use and application of smell in the automotive context.

\section{ACKNOWLEDGMENTS}

This work was supported by the EC within Horizon2020, through the ERC (Starting Grant Agreement 638605).

\section{REFERENCES}

1. R. A. Baron and M. J. Kalsher. 1998. Effects of a Pleasant Ambient Fragrance on Simulated Driving Performance: The Sweet Smell of... Safety? Env. and Beh. (1998).

2. A. Bodnar et al. 2004. AROMA: Ambient Awareness Through Olfaction in a Messaging Application. In ICMI '04. ACM, New York, NY, USA.

3. S. Brewster et al. 2006. Olfoto: Designing a Smell-based Interaction. In $\mathrm{CHI}$ '06. ACM, New York, NY, USA.

4. R. S. Herz and T. Engen. 1996. Odor memory: Review and analysis. Psychonomic Bul. \& Rev. (1996).

5. J. Koo et al. 2015. Why did my car just do that? Explaining semi-autonomous driving actions to improve driver understanding, trust, and performance. In IJIDeM.

6. G. N. Martin and J. A. Cooper. 2007. Adding zest to difficult journeys: Odour effects on simulated driving performance. In BPS Annual Conference.

7. M. Obrist et al. 2014. Opportunities for Odor: Experiences with Smell and Implications for Technology. In CHI '14. ACM, New York, NY, USA.

8. B. Pfleging et al. 2012. Multimodal Interaction in the Car: Combining Speech and Gestures on the Steering Wheel. In AutomotiveUI '12. ACM, New York, NY, USA.

9. B. Raudenbush et al. 2009. Effects of Peppermint and Cinnamon Odor Administration on Simulated Driving Alertness, Mood and Workload. North American J. of Psychology (2009).

10. P. Sundström et al. 2014. Gaming to Sit Safe: The Restricted Body As an Integral Part of Gameplay. In DIS '14. ACM, New York, NY, USA.

11. S. Trösterer et al. 2014. Using a Parking Assist System Over Time: Insights on Acceptance and Experiences. In AutomotiveUI '14. ACM, New York, NY, USA.

12. D. Warnock et al. 2013. Multiple Notification Modalities and Older Users. In CHI '13. ACM, New York, NY, USA.

13. T. Weiss et al. 2012. Perceptual convergence of multi-component mixtures in olfaction implies an olfactory white. National Academy of Sciences (2012).

14. M. Yoshida et al. 2011. Study on Stimulation Effects for Driver Based on Fragrance Presentation. In IAPR Conference on Machine Vision Appl., Nara, Japan. 